\title{
Impaired mitophagy and protein acetylation levels in fibroblasts from Parkinson's disease patients
}

Sokhna M.S. Yakhine-Diop $\mathrm{PhD}^{1,2}$, Mireia Niso-Santano $\mathrm{PhD}^{1,2}$, Mario RodríguezArribas $\mathrm{MSc}^{1,2}$, Rubén Gómez-Sánchez $\mathrm{PhD}^{3}$, Guadalupe Martínez-Chacón $\mathrm{PhD}^{1,2}$, Elisabet Uribe-Carretero MSc${ }^{1,2}$, José A. Navarro-García $\mathrm{MSc}^{4}$, Gema Ruiz-Hurtado $\mathrm{PhD}^{4}$, Ana Aiastui $\mathrm{PhD}^{2,5,6}$, J.M. Cooper $\mathrm{PhD}^{7}$, Adolfo López de Munain $\mathrm{PhD}^{2,6,8,9,10}$,

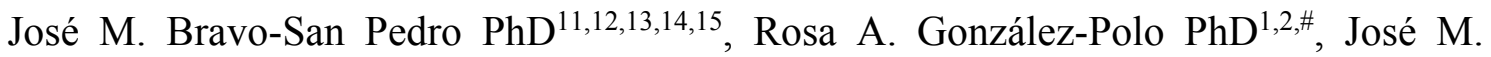
Fuentes $\mathrm{PhD}^{1,2, \#}$

${ }^{1}$ Universidad de Extremadura. Departamento de Bioquímica y Biología Molecular y Genética. Facultad de Enfermería y Terapia Ocupacional. Avda. de la Universidad s/n, C.P. 10003 Cáceres. ${ }^{2}$ Centro de Investigación Biomédica en Red en Enfermedades Neurodegenerativas (CIBERNED) Madrid, Spain. ${ }^{3}$ Department of Cell Biology, University of Groningen, University Medical Center Groningen, A. Deusinglaan 1, 9713 AV Groningen, The Netherlands. ${ }^{4}$ Laboratorio de Hipertensión y Riesgo Cardiovascular and Unidad de Hipertensión, Instituto de Investigación imas12, Hospital Universitario 12 de Octubre; Madrid, Spain. ${ }^{5}$ Cell Culture Plataform, Donostia University Hospital, San Sebastián, Spain. ${ }^{6}$ Neuroscience Area of Biodonostia Health Research Institute, Donostia University Hospital, San Sebastián, Spain. ${ }^{7}$ Department of Clinical Neurosciences, University College London, Institute of Neurology London, UK. ${ }^{8}$ Department of Neurology, Donostia University Hospital, San Sebastian, Spain; 9 Ilundain Fundazioa, San Sebastian, Spain; ${ }^{10}$ Department of Neurosciences, University of the Basque Country UPV-EHU, San Sebastián, Spain. ${ }^{11}$ Equipe 11 labellisée Ligue contre le Cancer, Centre de Recherche des Cordeliers, 75006 Paris, France. ${ }^{12}$ INSERM U1138, 75006 Paris, France. ${ }^{13}$ Université Paris Descartes/Paris V, Sorbonne Paris Cité, 75006 Paris, France. ${ }^{14}$ Université Pierre et Marie Curie/Paris VI, 75006 Paris, France. ${ }^{15}$ Gustave Roussy Comprehensive Cancer Institute, 94805 Villejuif, France.

\# These authors contributed equally.

Corresponding author: Centro de Investigación Biomédica en Red en Enfermedades Neurodegenerativas (CIBERNED), Departamento de Bioquímica y Biología Molecular Molecular y Genética. Facultad de Enfermería y Terapia Ocupacional. Universidad de Extremadura. Avda. De la Universidad S/N, C.P. 10003 Cáceres (Spain). Tel.: +34 927257450; Fax:+34927257451. E-mail address: jfuentes@unex.es; rosapolo@unex.es

Keywords: Acetylation/Histone acetyltransferases/Histone deacetylases/LRRK2/ mitophagy.

\section{Acknowledgments:}

We are grateful to the patients and donors without whom this work would not have been possible. The authors thank M. P. Delgado-Luceño, J.A. Tapia-Garcia. SMS.Y-D was supported by Isabel Gemio Foundation. M. N-S was funded by "Ramon y Cajal 
Program (RYC-2016-20883) Spain. M. R-A. and E. U-C were supported by a FPU predoctoral fellowship (FPU13/01237 and FPU16/00684, respectively) from Ministerio de Educación, Cultura y Deporte, Spain. R. G-S. was supported by a Marie Sklodowska-Curie Individual Fellowship (IF-EF) (655027) from the European Commission. JM. B-S. P. was funded by La Ligue Contre le Cancer. JM. F. received research support from the Instituto de Salud Carlos III, CIBERNED (CB06/05/004) and Instituto de Salud Carlos III, FIS, (PI15/00034). RA. G-P. was supported by a "Contrato destinado a la retención y atracción del talento investigador, TA13009" from Junta de Extremadura, and receiveed a research support from the Instituto de Salud Carlos III, FIS, (PI14/00170). JM.C. was funded by a Parkinson's UK project grant. This work was also supported by "Fondo Europeo de Desarrollo Regional" (FEDER) from the European Union. The authors also thank FUNDESALUD for helpful assistance. 


\begin{abstract}
:
BACKGROUND: Parkinson's disease (PD) is a chronic and progressive neurodegenerative disorder. While most PD cases are idiopathic, the known genetic causes of PD are useful to understand common disease mechanisms. Recent data suggests that autophagy is regulated by protein acetylation mediated by histone acetyltransferase (HAT) and histone deacetylase (HDAC) activities. The changes in histone acetylation reported to be involved in PD pathogenesis have prompted this investigation of protein acetylation and HAT and HDAC activities in both idiopathic PD and G2019S leucine-rich repeat kinase 2 (LRRK2) cell cultures.
\end{abstract}

METHOD: Fibroblasts from PD patients (with or without the G2019S LRRK2 mutation) and control subjects were used to assess the different phenotypes between idiopathic and genetic PD.

RESULTS: G2019S LRRK2 mutation displays increased mitophagy due to the activation of class III HDACs whereas idiopathic PD exhibits downregulation of clearance of defective mitochondria. This reduction of mitophagy is accompanied by more reactive oxygen species (ROS). In parallel, the acetylation protein levels of idiopathic and genetic individuals are different due to an upregulation in class I and II HDACs. Despite this upregulation, the total HDAC activity is decreased in idiopathic $\mathrm{PD}$ and the total HAT activity does not significantly vary.

CONCLUSION: Mitopahgy upregulation is beneficial for reducing the ROS-induced harm in genetic PD. The defective mitophagy in idiopathic PD is inherent to the decrease in class III HDACs. Thus, there is an imbalance between total HATs and HDACs activities in idiopathic PD, which increases cell death. The inhibition of HATs in idiopathic PD cells displays a cytoprotective effect. 


\section{Introduction}

Parkinson's disease (PD) is characterized by an idiopathic disorder with a multifactorial origin in which genetic susceptibility combined with environmental factors can trigger disease onset. The most prevalent gene mutation is G2019S LRRK2, which is present in $4 \%$ of familial PD and 1\% of sporadic PD [1]. Environmental factors classified as pesticides (dieldrin, paraquat) [2-4], and neurotoxins (1-methyl-4-phenylpyridinium iodide $\left.\left[\mathrm{MPP}^{+}\right]\right)[5]$ have been shown to inhibit mitochondrial function and lead to the induction of reactive oxygen species (ROS) and ultimately cell death.

Autophagy removes damaged organelles (e.g., mitochondria) to maintain cellular homeostasis. Altered mitochondria are cleared by mitophagy, a selective type of autophagy that is mediated by the PTEN-induced putative kinase 1 (PINK1)/Parkin pathway $[6,7]$. Impairment of autophagy has been reported in PD models and might worsen the progression of PD pathogenesis [8]. Autophagy is a complex mechanism that is widely regulated through the mammalian target of rapamycin (mTOR) or AMPactivated protein kinase (AMPK) signaling [9] or acetylation [10]. The latter intervenes downstream of the mTOR pathway [10]. Histone acetyltransferases (HATs) and histone deacetylases (HDACs) mediate the addition and the removal of acetyl group from lysine (K) residues of proteins, respectively. Their activity induces post-translational modifications of proteins, including histones that epigenetically regulate gene expression $[11,12]$. Two classes of HATs exist: class A (p300/ CREB-binding protein (CBP), GCN5-related N-acetyltransferase (GNAT), MYST families) and class B, which correspond to nuclear and cytosolic HATs, respectively [11]. Their antagonists, HDACs, are classified into four groups (supplementary Table 1). Class I, II and IV HDACs are $\mathrm{Zn}^{2+}$-dependent [13], while class III HDACs, (SIRTs) are NAD ${ }^{+}$-dependent [12].

HDACs/HATs have been reported to be important at different stages of autophagy regulation $[10,14]$, including the initiation and elongation of phagophore formation. The HDAC SIRT1 and the HAT p300 proteins control this mechanism through the regulation of acetylation of autophagy-related (ATG) proteins, including ATG5, ATG7 and ATG8/light-chain microtubule-associated protein (LC3) $[15,16]$. However, not all ATG proteins are deacetylated for autophagy induction; ATG3 and ATG1/unc-51-like kinase 1 (ULK1) are acetylated by tat-interactive protein 60 (TIP60) HAT [10, 14]. ATG are essential throughout the process of phagophore formation leading to the autophagosome [17]. Starvation leads to hypoacetylation of a variety of ATG [15] and cytosolic proteins [18], which may occur due to decreased interactions with HAT proteins [16].

Evidence has implicated epigenetic modifications in the progression of neurodegenerative diseases due to their ability to change gene expression and cellular phenotype $[19,20]$. Modified histone acetylation levels have been shown in distinct PD models, although existing results are inconclusive. The translocation of $\alpha$-synuclein into the nucleus [21] is associated with the hypoacetylation of histones and promotes 
apoptotic cell death [22]. Based on this hypoacetylation, class I and II HDACs inhibitors were found to be neuroprotective [22, 23]. However, in other PD models, protein acetylation was increased. In dopaminergic cells, while paraquat increased the acetylation of histone 3 (H3) but not histone 4 (H4) [20], dieldrin enhanced both acetylated $\mathrm{H} 3$ and $\mathrm{H} 4$ [4]. Unexpectedly, paraquat inhibited HDAC activity [20], whereas dieldrin augmented the level of CBP protein [4]. Thus, HATs/HDACs participate in the pathogenesis of PD by regulating cell survival and/or cell death in response to stimuli. Herein, under basal conditions, we characterized the acetylation protein levels in fibroblasts from idiopathic PD patients and familial G2019S LRRK2 mutation. We highlight the intricate role of HATs and HDACs in protein acetylation, mitophagy impairment and cell death.

\section{Materials and Methods}

\section{Cellular models}

Experiments were performed using three fibroblast groups: Control (Co, subjects who did not develop PD), idiopathic PD (IPD, PD patients without G2019S LRRK2 mutation) and GS (PD patients with G2019S LRRK2 mutation). Each group is a pool of three to four cell lines (supplementary Table 2). This study was performed in agreement with the Comité Ético de Investigación Clínica del Área Sanitaria de Gipuzkoa. All subjects gave written informed consent in accordance with the Declaration of Helsinki. Human fibroblasts (HF) were maintained as previously described [24]. To determine G2019S LRRK2 mutation, DNA was extracted (Macherey-Nagel, 740952.50), the LRRK2 exon 41 amplified (DreamTaq Master Mix, ThermoFisher, K1071 with two oligonucleotides 630028776-77 from IDT®) and digested by the restriction enzyme BfmI/SfcI (ThermoFisher, ER1161) (Fig. S1A). We also used SH-SY5Y neuroblastoma cells that stably overexpress WT LRRK2 (WT) or G2019S LRRK2 (G20) [25] to highlight the differences between neurons and fibroblasts.

Cells were treated with anacardic acid (AA, A72336), carbonyl cyanide 3chlorophenylhydrazone (CCCP, C2759), cyclosporin A (CsA, C3662), EX-527 (E7034), Earl's Balanced Salt Solution (EBSS, for autophagy induction, E2888), nicotinamide (NAM, N0636), $\mathrm{MPP}^{+}$(D048), and trichostatin A (TSA, T8552) from Sigma-Aldrich and bafilomycin A1 (BAF. A1, LC Laboratories, B-1080).

\section{Cell viability}

Cell viability was assessed via a 3-(4,5-dimethylthiazol-2-yl)-2,5-diphenyltetrazolium bromide (MTT, Sigma-Aldrich, M2128) assay. Cells were treated and then incubated with MTT $(0.45 \mathrm{mg} / \mathrm{mL})$ [26]. The formazan precipitate was solubilized in isopropanol acid and the absorbance was read at $570 \mathrm{~nm}$ using the microplate reader (TECAN Sunrise). 


\section{Western-blotting analysis}

For whole-cells extract, cells were lysed in buffer containing 0.5\% NP-40 (Roche, 11754599001), $100 \mathrm{mM}$ Tris- $\mathrm{HCl}(\mathrm{pH} 7.4), 300 \mathrm{mM} \mathrm{NaCl}$ and complemented with protease and phosphatase inhibitors [27], AA $(10 \mu \mathrm{M})$ and HDAC inhibitor cocktail (Santa-Cruz, sc-362323). Proteins were resolved by SDS-gel electrophoresis and blots probed with antibodies against acetylated-K (Ac-K) (\#9441), DRP1 (D6C7) (\#8570), H3 (\#9715), acetyl-H3K14 (\#4318, Ac-H3K14 (D4B9), \#7627), PCAF (C14G9) (\#3378), SIRT1 (D1D7) (\#9475), pSIRT1 (serine 47) (\#2314), and acetyl- $\alpha$-tubulin (K40) (Ac-TubK40, \#3971) from Cell Signaling, acetyl-H4Ser1K5K8K12 (G-2) (AcH4K5K8K12, sc-393472), HDAC6 (H-300) (sc-11420), hMOF (G-12) (sc-271691), p300 (F-4) (sc-48343), TOM20 (F-10) (sc-17764), a-tubulin (TU-02) (sc-8035) from Santa Cruz, GAPDH (Millipore, NG1740950), LC3 (, L7543) and SIRT1 (S5322) from Sigma-Aldrich, LONP1 (Proteintech, 15440-1-AP), subunit IV of cytochrome c oxidase (COX IV, Abcam, ab14744), PINK1 (Novus biologicals, BC100-494) and TIP60 (Calbiochem, DR1041).

\section{Flow cytometry assay}

Cells were preloaded with MitoTracker ${ }^{\circledR}$ Green FM (MTG, ThermoFisher, M7514) and washed with PBS before treatment [28]. Treated cells were detached by trypsin-EDTA (Sigma-Aldrich, T4049), collected in FACS tubes and loaded with $200 \mu \mathrm{L}$ MTG solution (100 nM in complete medium) for $15 \mathrm{~min}$ at $37^{\circ} \mathrm{C}$ to monitor mitochondrial mass. Subsequently, propidium iodide $(0.1 \mathrm{mg} / \mathrm{mL})$ (PI, Sigma-Aldrich, P4170) was added to each tube to detect the percentage of cell death (or PI-positive cells). Cells were also stained with Annexin V-FITC (Immunostep, ANXVF-200T) to examine the percentage of apoptotic cells. Stained cells were analyzed by flow cytometer (Beckman Coulter FC500-MPL).

\section{Mitochondrial membrane potential (MMP) and ROS detection}

MMP was evaluated by the green fluorescent probe $\operatorname{DiOC}_{6}(3) \quad\left(3,3^{\prime}-\right.$ dihexyloxacarbocyanine iodide) (Invitrogen, D273) that is retained in the inner membrane of healthy mitochondria. During cell death, mitochondria fail to retain the fluorochrome, and cells become $\operatorname{DiOC}_{6}(3)$ deficient. MMP is reduced in defective mitochondria, and the level of ROS increases. We measured ROS levels by detecting the accumulation of superoxide with dihydroethidium (Invitrogen ${ }^{\mathrm{TM}}$, D1168) or MitoSOX $^{\mathrm{TM}}$ Red (Invitrogen ${ }^{\mathrm{TM}}$, M36008) for specific mitochondrial ROS. In presence of superoxide, both dihydroethidium and MitoSOX ${ }^{\mathrm{TM}}$ are oxidized to ethidium and emits red fluorescence [29]. The percentages of $\mathrm{DiOC}_{6}(3)$-deficient and ethidiumpositive cells were determined by flow cytometer.

\section{HAT activity colorimetric assay}


The total activity of HATs was detected using a HAT colorimetric kit (Biovision, \#K332-100) according to the manufacturer' instructions. An amount of $50 \mu \mathrm{g}$ proteins from the whole cell lysate was mixed with acetyl-CoA, the peptide substratetetrazolium dye and the generating NADH enzyme. Samples were incubated at $37^{\circ} \mathrm{C}$ for $3 \mathrm{~h}$. During the acetylation of peptide, CoA is released to produce NADH with the generating enzyme. The NADH-tetrazolium reduction is detected at $400 \mathrm{~nm}$ using the plate reader (Asys UVM 340). HAT activity is represented as the absorbance/ $\mu \mathrm{g}$ protein.

\section{HDAC activity colorimetric assay}

The total activity of HDACs was evaluated using a HDAC colorimetric kit (Biovision, \#K331-100) according to the manufacturer' instructions. $130 \mu \mathrm{g}$ proteins from the whole cell lysate were mixed with HDAC substrates during $1 \mathrm{~h}$ at $37^{\circ} \mathrm{C}$. After that, a solution of lysine developer is added to the mixture during $30 \mathrm{~min}$ at $37^{\circ} \mathrm{C}$ to detect the quantity of deacetylated lysine. The absorbance of the developed chromophores in samples was read at $405 \mathrm{~nm}$ using the plate reader Asys. HDAC activity is represented as the absorbance/ $\mu \mathrm{g}$ protein.

\section{Immunofluorescence microscopy}

Cells were seeded on 96-well plate. After treatment, cells were fixed with 4\% PFA and permeabilized (except for the determination of cytoplasm Ac-K proteins [30]) with $0.1 \%$ Triton (Sigma-Aldrich, T9284). Once permeabilized, cells were incubated with bovine serum albumin (BSA)/PBS solution $(1 \mathrm{mg} / \mathrm{mL})$ for $1 \mathrm{~h}$ and then with Ac-K (AKL5C1) (Santa Cruz, sc-32268), TOM20 (F-10) and Ac-H4K16 (E2B8W) (Cell Signaling, \#13534) antibodies overnight at $4^{\circ} \mathrm{C}$. Thereafter, cells were reincubated with ThermoFisher Alexa Fluor ${ }^{\circledR} 568$ (A11004) or 488 (A11008)-conjugated secondary antibodies for $1 \mathrm{~h}$ at RT. Nuclei were stained with Hoechst 33342 (2 $\mu \mathrm{M}$, SigmaAldrich, B2261). Cells were loaded $45 \mathrm{~min}$ before the end of the treatment with $50 \mathrm{nM}$ Tetramethylrhodamine, methyl ester, perchlorate (TMRM, Molecular probes, T668) at $37^{\circ} \mathrm{C}$ to determine drug effects on MMP. Images were visualized using an Olympus IX51 inverted microscope equipped with a DP71 camera.

\section{Mitochondrial extraction}

Mitochondria were isolated using a Mitochondria Isolation Kit (ThermoFisher, 89874); the option A of the manufacturer's instructions was selected. The required amounts of reagent $\mathrm{A}$ and reagent $\mathrm{B}$ were supplemented with protease inhibitor cocktail 10X (Sigma-Aldrich, P2714), 0.5 M 20\% sodium orthovanadate (Sigma-Aldrich, S6508), and $0.1 \mathrm{M} 1 \%$ sodium fluoride (Panreac, 131675). Isolated mitochondria were lysed in 2\% CHAPS (Sigma-Aldrich, C3023). Cytosolic and mitochondrial extractions were analyzed by Western-blotting (WB).

\section{Plasmid transfection}


HFs were plated on uncoated coverslip and transiently transfected with LC3-GFP constructs $(3 \mu \mathrm{g} / 100 \mu \mathrm{L})$ (gift of Dr. Tamotsu Yoshimori) using Lipofectamine ${ }^{\mathrm{TM}} 2000$ transfection reagent $(3 \mu \mathrm{L} / 100 \mu \mathrm{L})$ (Invitrogen, 11668027) [31]. Cells were fixed with PFA and coverslips were mounted on the slides using ProLong ${ }^{\circledR}$ Diamond Antifade Mountant with DAPI (ThermoFisher, P36966). Images were visualized using a Carl Zeiss-LSM510 laser confocal microscopy. LC3 puncta were analyzed using Ifdotmeter ${ }^{\circledR}$ software [32], and the colocalization data were assessed with the JACoP plugin of ImageJ [33].

\section{Quantitative RT-PCR}

Total RNA was extracted using an RNeasy mini kit (Qiagen, \#CAT 74104) and then reverse-transcribed into complementary DNA (cDNA) via QuantiTect reverse transcription (Qiagen, 205311). The cDNA was amplified by qPCR with a KAPA SYBR ${ }^{\circledR}$ Fast kit (Kapa Biosystems, KK4601) using the following primers from IDT®: GAPDH (68815248-49), HDAC1 (65776039-40), HDAC2 (65776041-42), HDAC3 (65776043-44), HDAC4 (65776045-46), HDAC6 (65776047-48), HDAC7 (6577604950), hMOF (65776071-72), p300 (65776061-62), PCAF (65776063-64), TIP60 (65776073-64), SIRT1 (65776051-52), SIRT2 (65776053-54), SIRT3 (65776055-56), SIRT5 (65776057-58), and SIRT6 (65776059-70). GAPDH gene expression was used as an endogenous control, and expression levels were determined by the $2^{(-\Delta \Delta \mathrm{Ct})}$ ratio [34].

\section{RNA interference:}

Genetic inhibition of SIRT1 was performed by Hiperfect transfection reagent (Qiagen, Lot No 148035183) and the human SIRT1 siRNA (Ambio, Cat AM16708, ID 19833) at $10 \mathrm{nM}$. We also used the negative control siRNA (Ambion, L/N 1602012) at the same concentration.

\section{Statistical analyses}

Data represent the mean \pm SD or mean \pm SEM of at least three experiments. Statistical analyses were assessed by Student's t tests. The results were considered significant at $\mathrm{p}<0.05$.

\section{Results:}

\section{Impaired mitophagy flux in fibroblasts from PD patients}

To assess mitophagy in fibroblasts from PD patients, cells were transfected with GFPLC3 plasmid and co-labeled with the outer mitochondrial membrane protein TOM20. We detected increased co-localization of TOM20 with GFP-LC3 puncta in both IPD and GS cells (Fig. 1A, B). Consistent with that, the number of GFP-LC3 puncta was significantly increased in PD lines (Fig. 1C). Our group previously demonstrated that autophagy is exacerbated and efficiently degradative in GS cells [35]. Measuring basal 
autophagic flux of IPD cells treated with EBSS or with the lysosomal blocker BAF. A1 displayed an augmentation of LC3-II (Fig. 1D, E). The difference in LC3-II between Co and IPD cells was significant under basal and starvation conditions but not with BAF.A1 condition. To evaluate mitochondrial degradation, we treated cells with CCCP, a mitochondrial uncoupler that fosters mitophagy [36], and/or the mitophagy inhibitor CsA [37]. Under basal conditions (Fig. 1F), IPD cells exhibited a higher percentage of MTG fluorescence than Co cells, which is shown in Fig. $1 \mathrm{G}$ by a shift of the red histogram to the right, whereas GS presented a lower \% of MTG, which was characterized by a displacement of the green histogram to the left. Thus, mitochondrial content was significantly increased in IPD and reduced in GS cells (Fig. 1F). CCCP promoted the shift of the purple histogram to the left for Co cells (Fig. 1H), and decreased significantly the percentage of MTG fluorescence in IPD cells (Fig. 1F). Mitochondrial content was remarkably increased with CsA in all fibroblasts (Fig. 1F). These results suggest that, under basal conditions, mitophagy was reduced and increased in IPD and GS cells, respectively. Moreover, we observed a MMP decrease (Fig. 2A), a ROS generation (Fig. 2B) and a mitochondrial ROS production (Fig. 2C) in PD fibroblasts. Other mitochondrial markers were studied, including LON peptidase 1 (LONP1), a mitochondrial matrix protease that reduces PINK1 accumulation and dynamin-related protein 1 (DRP1). A reduction of LONP1 induces mitophagy by increasing PINK1 accumulation [6]. Our results showed that LONP1 protein was reduced in PD cells (Fig. 2D, E), which indicated that the early stage of mitochondrial degradation signaling (at least the PINK1/Parkin pathway) was not perturbed (Fig. 2G). Furthermore, DRP1, which induces mitochondrial fission processes [38], was upregulated in both PD-associated fibroblasts (Fig. 2D, F) and mitochondria were also fragmented (Fig. 2H). Mitophagy induction is promoted in both PD cells, however, it seems to be reduced in IPD cells at basal level.

\section{Autophagy modulation by sirtuins}

SIRTs play a critical role in mitochondrial function and cell survival in neurodegenerative disorders [39]. We considered the possibility that variations in SIRTs expression may cause impaired mitophagy in PD cells. Interestingly, mRNA expression levels of SIRTs (Fig. 2I, L, M), except SIRT2 (Fig. S1B), were significantly decreased in IPD cells. SIRT5 (Fig. 2M) and SIRT6 (Fig. S1F) behaved equally in PD fibroblasts. Our result revealed an increase in the phosphorylation of SIRT1 in GS cells (Fig. 2J, K). In addition, SIRT3 protein level decreased in PD fibroblasts (Fig. 2O, Q). In contrast to GS cells, IPD cells exhibited augmented SIRT5 (Fig. 2N, P) and TOM20 (Fig. 3A) protein levels. SIRT3 is exclusively a mitochondrial protein and SIRT5 is both cytosolic and mitochondrial protein [40]. If mitophagy is activated in GS cells [41], dopaminergic neurons expressing G2019S LRRK2 mutation (G20 cells) must display a reduction of mitochondrial markers. Consistent with our hypothesis, SIRT3 and SIRT5 as well as TOM20 and COX IV proteins were decreased in G20 cells (Fig. 3B). However, CsA treatment increases SIRT3 and SIRT5 protein levels in PD cells (Fig. 3C). Accordingly, SIRT1 and SIRT2 (Fig. S1C-E), which are both nuclear and cytosolic proteins, and 
nuclear SIRT6 (Fig. S1G, H) were not reduced in GS cells. To further study the role of SIRTs in autophagy in GS cells, we treated cells with NAM, a nonspecific inhibitor of SIRTs. This experiment revealed that SIRTs inhibition (Fig. 3D-F) decreased ATG5 protein and LC3-II levels in GS cells towards the Co protein levels. The same result was observed (Fig. 3G-I) for both ATG5 and LC3-II protein levels with EX-527 treatment, a SIRT1-specific inhibitor. Additionally, EX-527 (Fig. 3J) or the genetic inhibition of SIRT1 (Fig. 3K) increased the TOM20 protein level in GS cells. In Fig. 3L, we can observe that SIRT1 inhibition induces a mitochondrial fragmentation. Together, SIRT expression is generally reduced in IPD models and SIRT1 is involved in mitophagy/autophagy regulation in GS cells.

\section{Acetylated protein levels in cellular models of PD}

Given that SIRT expression varies among PD models, the level of Ac-K proteins was evaluated. Fig. 4A shows that there were two populations of Ac-K proteins in PD models. In HFs, proteins in the range of $15-25 \mathrm{kDa}$ were less acetylated; however, higher molecular weight $(37-250 \mathrm{kDa})$ proteins were more acetylated. Moreover, the level of Ac-K proteins in the range of 100-250 kDa was increased in GS cells, whereas it was reduced in IPD cells. Similar to that observed in GS cells, proteins were not hypoacetylated in G20 cells despite the minimal load of total proteins. Next, we revealed the intensity of acetylated cytosolic and nuclear proteins. In Fig. 4B, C and Fig. S2A, B, G, GS cells clearly exhibited a higher intensity of Ac-K proteins than Co and IPD cells. Thus, SIRTs did not affect the level of Ac-K proteins in GS cells. Although autophagy was activated [35] in cells harboring the G2019S LRRK2 mutation, Ac-K protein levels were decreased during starvation-induced autophagy (Fig. 4D-F). Thereafter, we investigated the expression of four HATs in Ac-K proteins differences observed between PD models. Surprisingly, none of them were enhanced in GS (Fig. S2) and G20 cells (Data not shown). p300 (Fig. S2C) did not vary [42] and PCAF (Fig. S2D) was remarkably reduced in GS cells. hMOF (Fig. S2E) and TIP60 (Fig. S2F), were significantly decreased in PD models. In GS cells, the important diminution of TIP60 and PCAF mRNA expression did not alter their protein levels (Fig. 4G, H and Fig. 4J, K, respectively). In contrast, TIP60 protein was significantly reduced in IPD cells (Fig. 4G, H). We were unable to detect p300 and hMOF proteins by WB because the purchased antibodies failed. Therefore, we assessed HAT activity. Although PD models exhibited increased HAT activity (Fig. 4I), it was not remarkable, possibly because we did not distinguish between nuclear HATs and cytoplasmic HATs. We next sought to examine substrates of HATs, such as H4 and H3. The antibody used recognizes $\mathrm{H} 4$ acetylated at $\mathrm{K} 5, \mathrm{~K} 8$, or $\mathrm{K} 12$. If all lysine residues were acetylated, AcH4K5K8K12 would acquire a molecular weight of $35 \mathrm{kDa}$. We found a band of $11 \mathrm{kDa}$, indicating that $\mathrm{H} 4$ was acetylated at one $\mathrm{K}$, and its level was strikingly reduced in IPD cells (Fig. 4L, M). p300 activity is required for Ac-H4K5K8K12, and PCAF activity is required for Ac-H4K8 [43]. At least one of those residues was acetylated in Co and GS cells compared with IPD cells, and we therefore inferred that PCAF or p300 was 
activated in GS cells. Interestingly, while nuclear proteins were less acetylated in IPD cells, cytoplasmic protein ( $\alpha$-tubulin) was significantly acetylated at K40 (Fig. 4N, O).

\section{Decreased HDAC activity in PD}

To gain further insight into the differences in acetylation levels between PD models, we assessed HDAC activity by colorimetric essay and HDAC expression. The mRNA expression levels of HDACs (Fig. S3) did not significantly differ between lines except HDAC1 and HDAC4 in GS cells. When we evaluated the protein expression, an increasing level of class I HDAC proteins (HDAC2 and HDAC3) was observed in IPD cells (Fig. 5A-D), whereas only HDAC2 was enhanced in GS cells. Class II HDACs were conversely modulated in PD cells. While class IIa (HDAC4) was significantly increased (Fig. 5E, F) in PD models, class IIb (HDAC6) was downregulated (Fig. 5H, I). These experiments suggest that there are relevant expression levels of Class I and IIa HDACs in fibroblasts of PD patients. Therefore, we deduced that HDAC3 protein might underlie the difference between PD cells, as HDAC1 did not fluctuate (Fig. 5E, G). Furthermore, HDAC3 is a nuclear and cytosolic protein but it is more localized in the nucleus [44]. Despite the upregulation of HDAC proteins in IPD cells, HDAC activity (HADCs/SIRTs) (Fig. 5J) was remarkably diminished, and this reduction was not significant in GS and G20 cells.

\section{Cytotoxic effect of HDAC inhibitors}

Given that the attenuation of HDAC activity did not correspond to the reduced level of Ac-K proteins in IPD cells, we examined the effect of HDACs and SIRTs in parallel. Cells were treated with non-selective inhibitors of class I and II HDACs (TSA) [45] or class III HDACs (NAM) [46]). HFs were more susceptible to NAM than TSA during a dose-response treatment (Fig. S4A, B). Consequently, we used lower concentrations of TSA $(1 \mu \mathrm{M})$ and NAM $(1 \mathrm{mM})$ that could inhibit HDAC activity. TSA potentiated the intensity of Ac-H3K14, which generally masked the modest effect of both NAM-treated and untreated cells. Nevertheless, we observed that NAM did not enhance Ac-H3K14 in IPD cells (Fig. S4C, D). In addition, the intensity of Ac-H4K16 was increased with TSA compared with NAM or untreated cells (Fig. 5K). More class I and II HDAC activity appeared to be present in cell lines, or the used concentration of NAM was not sufficiently potent for SIRT inhibition. To verify this hypothesis, we investigated the effect of HDAC inhibitors on PD-related cell death. We observed that the percentage of apoptotic cells dramatically increased in PD cells compared with Co cells. In addition, TSA or NAM treatment elicited cell death in healthy controls and did not exert a protective effect in PD models (Fig. 5L). In fact, compared with CCCP [36], HDAC inhibitors decreased the MMP (Fig. 5M, N). Taken together, the concentration of NAM was effective, and cells were susceptible to SIRT inhibition (Fig. 5L), even though there was less SIRT activity than HDAC activity in cell lines.

\section{Protective effect of HAT inhibitor}


Histones were hyperacetylated in IPD cells when class I and II HDACs were inhibited. We questioned whether HATs were involved in the acetylation of nuclear proteins. Consequently, cells were treated with AA, a potent inhibitor of HATs, including PCAF, p300 [47] and TIP60 [4]. We decided to detect the level of Ac-H3K14, a common substrate of PCAF [48] and p300 [43]. We observed that there was less Ac-H3K14 in IPD cells than in Co and GS cells (Fig. 6A, B). This level was reduced in all cell lines treated with AA but it was not significantly different between treated and untreated GS cells. Our results suggest that HAT proteins are activated in PD models, and the level of Ac-H3K14 becomes significant, between Co and GS cells, with AA. Moreover, the inhibition of HATs had a protective effect on IPD cells and not on GS cells (Fig. 6C). To further elucidate this positive response, we reproduced an oxidative stress condition by treating WT cells with $\mathrm{MPP}^{+}$and/or AA overnight. With A. A treatment alone, the same results were observed in neurons (Fig. 6D) and fibroblasts Co (Fig. 6C). Additionally, AA reduced $\mathrm{MPP}^{+}$-induced cell death (Fig. 6D).

\section{Discussion}

Autophagy impairment and protein acetylation status are associated with PD pathogenesis. Furthermore, autophagy is regulated by HDAC/HAT enzymes, which remove and replace the acetyl group on lysine of proteins, respectively. Autophagy is degradative in GS fibroblasts through the ERK1/2 signaling pathway [35]. In this study, we found that IPD fibroblasts displayed a decreased basal autophagy/mitophagy (Fig. 1D and Fig. 1F). As SIRT1 was reported to deacetylate ATG proteins [15], and SIRT1 was phosphorylated in GS cells (Fig. 2J), the implication of class III HDACs becomes obvious regarding this difference in mitophagy flux between IPD and GS cells. SIRT1 phosphorylation promotes its translocation into the nucleus, where LC3 is deacetylated prior to the initiation of autophagosome formation [49]. The inhibition of SIRT1 prevented LC3 lipidation and increased TOM20 in GS cells (Fig. 3). If SIRT1 is activated in our model, it may be due either to the level of ROS production (Fig. 2B, C) or activation of the ERK1/2 pathway [35]. The MAPK pathway is activated in response to cellular stress and modulates SIRT1 function. JNK1 enhances SIRT1 activity by phosphorylation of serine 47 [50], and SIRT1 activates the ERK1/2 pathway [51]. The study of Zhao et al. supports the idea that ERK1/2 activates SIRT1 and that SIRT1 can activate ERK1/2 [52]. We did not demonstrate a direct interaction between ERK1/2 and SIRT1 in GS cells. However, while the inhibition of ERK1/2 reduced cell death in GS cells [35], the inhibition of SIRT1 (Fig. S4E) was not protective, and these data are consistent with Zhao's previous report [52]. We believe that GS cells attempt to resist stress-induced apoptosis due to SIRT1 activation, and the difference in SIRT1 activity between IPD and GS may explain the differences in apoptotic cell death levels between these two cells lines. If oxidative stress regulates SIRT1 activity, then the process that is responsible for SIRT1 phosphorylation in GS cells, and not in IPD cells, is unclear.

ROS production is inherent to the accumulation of damaged mitochondria. Regardless of whether basal mitophagy is increased in PD models, DRP1 upregulation is accompanied by apoptotic cell death concomitant to ROS production and mitochondrial 
membrane depolarization. Indeed, DRP1-deficient cells are resistant to CCCP-induced mitochondrial membrane depolarization [38]. PD fibroblasts were susceptible to EBSS and CCCP (Fig. 1), suggesting that IPD cells have the ability to eliminate the material to be degraded. Thereby, mitophagy impairment in IPD cells needs to be induced. On the other hand, mitochondrial SIRTs, SIRT3 and SIRT5, are critical for the reduction of ROS due to mitochondrial dysfunction. Both proteins regulate the expression of antioxidant enzymes [53-55]. In $\mathrm{H}_{2} \mathrm{O}_{2}$-treated cells, SIRT3 protein was upregulated and promoted mitochondrial biogenesis [53]. SIRT3 knockdown enhanced rotenoneinduced cell death and oxidative stress [55]. SIRT5 protein is upregulated by MPTP stimulation, but its inhibition accelerates the loss of MPTP-treated dopaminergic neurons in mice [54]. Curiously, SIRT5 is accumulated in IPD cells, and we questioned whether its accumulation is an adaptive response to chronic stress or due to mitophagy impairment. In contrast to IPD cells, GS and G20 cells exhibit degradation of SIRT3 and SIRT5 by effective mitophagy. Despite the reduction of both proteins, mitophagy seem to be beneficial for reducing ROS production in GS cells compared with IPD cells (Fig. 2B, C). As mitochondria are the main source of ROS generation, the lack of SIRT3 protein might be detrimental to the degradation of defective mitochondria in IPD cells. Of note, SIRT3 mRNA expression fluctuates during oxidative stress induction; it reaches a peak during the initial exposure and decreases thereafter [53]. In this regard, we speculate that the reduction in SIRT3 expression could be caused by chronic ROS levels in IPD.

This is the first time that a group of Ac-K proteins is assessed in cells from PD patients (Fig. 4A). Starvation-induced autophagy decreases the levels of cytosolic Ac-K proteins (Fig. 4D-F) by reducing acetyl-CoA levels [18]. In GS fibroblasts, the increasing level of Ac-K proteins did not prevent autophagy induction. How acetylated proteins are regulated during autophagy is unclear, as two autophagy inducers, rapamycin [30] and EBSS, distinctly affect Ac-K protein levels. We surmise that the most important factor is not the acetylation level of cytoplasm proteins, rather those of ATG proteins is indispensable. GS cells exhibited more Ac-K proteins than IPD cells [56], though their HAT activity was not significantly different (Fig. 4I), consistent with previous observations [57]. In neurodegenerative diseases, hypoacetylation is attributed to degradation of HAT proteins $[42,57]$. In our study, PCAF was significantly upregulated and could be related to the intense acetylation of some proteins observed in IPD cells. How histones are hypoacetylated in IPD cells remains unclear. Studies in vivo and in vitro on oxidative stress models of PD have demonstrated that acetylation levels are reduced with $\mathrm{MPP}^{+} / \mathrm{MPTP}$ and paraquat because of the increase in HDAC activity [58, 59]. In IPD cells, total HDAC activity was notably reduced, which was not correlated with the acetylation status of proteins. Consequently, supplemental inhibition with HDAC inhibitors was harmful. We conclude that there is an imbalance between total HAT and HDAC activities in IPD cells, which confers a cytoprotective effect to HAT inhibitor. Given that class I and IIa HDACs are upregulated in IPD cells, the reduction in total HDAC activity can be attributed to lower activity of class III HDACs. SIRTs are $\mathrm{NAD}^{+}$-dependent, and defective mitochondria affect the $\mathrm{NAD}^{+} / \mathrm{NADH}$ ratio [60]. 
Indeed, the mitochondrial complex I, which oxidizes NADH to $\mathrm{NAD}^{+}$is inhibited in IPD [61]. Therefore, SIRT activity is abrogated in IPD cells and leads to a compensatory increase in class I and II HDACs (Fig.6E) that are responsible for the hypoacetylation of proteins [56]. It would be interesting to explore whether specific inhibitors of class I HDACs [58] are beneficial in IPD cells.

Finally, our proposed mechanism is that damaged mitochondria reduce SIRT activity, which further affects mitophagy regulation. This damage results in an imbalance between HAT and HDAC activities in IPD. Therefore, idiopathic and genetic PD exhibit distinct epigenetic modifications that influence their gene expression and determine their phenotype due to the increase of class I and II HDACs. The inhibition of HATs or the activation of SIRTs may be excellent PD therapies. In the future prescribing specific treatments for PD patients would be of interest because the molecular regulation of each pathogenesis is unique. It is not well understood how the imbalance in HAT and HDAC activities is modulated, and future post-mortem studies of both idiopathic and genetic PD should be performed to elucidate these mechanisms.

\section{References:}

1. Lill, C.M., Genetics of Parkinson's disease. Mol Cell Probes, 2016. 30(6): p. 386-396.

2. Niso-Santano, M., et al., Activation of apoptosis signal-regulating kinase 1 is a key factor in paraquat-induced cell death: modulation by the Nrf2/Trx axis. Free Radic Biol Med, 2010. 48(10): p. 1370-81.

3. Gonzalez-Polo, R.A., et al., Paraquat-induced apoptotic cell death in cerebellar granule cells. Brain Res, 2004. 1011(2): p. 170-6.

4. Song, C., et al., Environmental neurotoxic pesticide increases histone acetylation to promote apoptosis in dopaminergic neuronal cells: relevance to epigenetic mechanisms of neurodegeneration. Mol Pharmacol, 2010. 77(4): p. 621-32.

5. Bernstein, A.I., et al., 6-OHDA generated ROS induces DNA damage and p53- and PUMA-dependent cell death. Mol Neurodegener, 2011. 6(1): p. 2.

6. Jin, S.M. and R.J. Youle, The accumulation of misfolded proteins in the mitochondrial matrix is sensed by PINK1 to induce PARK2/Parkin-mediated mitophagy of polarized mitochondria. Autophagy, 2013. 9(11): p. 1750-7.

7. Gomez-Sanchez, R., et al., PINK1 deficiency enhances autophagy and mitophagy induction. Mol Cell Oncol, 2016. 3(2): p. e1046579.

8. Gonzalez-Polo, R.A., et al., Inhibition of paraquat-induced autophagy accelerates the apoptotic cell death in neuroblastoma SH-SY5Y cells. Toxicol Sci, 2007. 97(2): p. 448-58. 
9. Gonzalez-Polo, R.A., et al., Is the Modulation of Autophagy the Future in the Treatment of Neurodegenerative Diseases? Curr Top Med Chem, 2015. 15(21): p. 2152-74.

10. Yi, C., et al., Function and molecular mechanism of acetylation in autophagy regulation. Science, 2012. 336(6080): p. 474-7.

11. Sterner, D.E. and S.L. Berger, Acetylation of histones and transcription-related factors. Microbiol Mol Biol Rev, 2000. 64(2): p. 435-59.

12. Costantini, S., et al., Genealogy of an ancient protein family: the Sirtuins, a family of disordered members. BMC Evol Biol, 2013. 13: p. 60.

13. Delcuve, G.P., D.H. Khan, and J.R. Davie, Roles of histone deacetylases in epigenetic regulation: emerging paradigms from studies with inhibitors. Clin Epigenetics, 2012. 4(1): p. 5.

14. Lin, S.Y., et al., GSK3-TIP6O-ULK1 signaling pathway links growth factor deprivation to autophagy. Science, 2012. 336(6080): p. 477-81.

15. Lee, I.H., et al., A role for the NAD-dependent deacetylase Sirt1 in the regulation of autophagy. Proc Natl Acad Sci U S A, 2008. 105(9): p. 3374-9.

16. Lee, I.H. and T. Finkel, Regulation of autophagy by the p300 acetyltransferase. J Biol Chem, 2009. 284(10): p. 6322-8.

17. Klionsky, D.J., et al., Guidelines for the use and interpretation of assays for monitoring autophagy (3rd edition). Autophagy, 2016. 12(1): p. 1-222.

18. Marino, G., et al., Regulation of autophagy by cytosolic acetyl-coenzyme A. Mol Cell, 2014. 53(5): p. 710-25.

19. Jowaed, A., et al., Methylation regulates alpha-synuclein expression and is decreased in Parkinson's disease patients' brains. J Neurosci, 2010. 30(18): p. 6355-9.

20. Song, C., et al., Paraquat induces epigenetic changes by promoting histone acetylation in cell culture models of dopaminergic degeneration. Neurotoxicology, 2011. 32(5): p. 586-95.

21. Goers, J., et al., Nuclear localization of alpha-synuclein and its interaction with histones. Biochemistry, 2003. 42(28): p. 8465-71. 
22. Kontopoulos, E., J.D. Parvin, and M.B. Feany, Alpha-synuclein acts in the nucleus to inhibit histone acetylation and promote neurotoxicity. Hum Mol Genet, 2006. 15(20): p. 301223.

23. Monti, B., et al., Valproic acid is neuroprotective in the rotenone rat model of Parkinson's disease: involvement of alpha-synuclein. Neurotox Res, 2010. 17(2): p. 130-41.

24. Yakhine-Diop, S.M., et al., G2019S LRRK2 mutant fibroblasts from Parkinson's disease patients show increased sensitivity to neurotoxin 1-methyl-4-phenylpyridinium dependent of autophagy. Toxicology, 2014. 324: p. 1-9.

25. Papkovskaia, T.D., et al., G2019S leucine-rich repeat kinase 2 causes uncoupling protein-mediated mitochondrial depolarization. Hum Mol Genet, 2012. 21(19): p. 4201-13.

26. Riss, T.L., et al., Cell Viability Assays, in Assay Guidance Manual, G.S. Sittampalam, et al., Editors. 2004: Bethesda (MD).

27. Rodriguez-Arribas, M., et al., Turnover of Lipidated LC3 and Autophagic Cargoes in Mammalian Cells. Methods Enzymol, 2017. 587: p. 55-70.

28. Mauro-Lizcano, M., et al., New method to assess mitophagy flux by flow cytometry. Autophagy, 2015. 11(5): p. 833-43.

29. Robinson, K.M., et al., Selective fluorescent imaging of superoxide in vivo using ethidium-based probes. Proc Natl Acad Sci U S A, 2006. 103(41): p. 15038-43.

30. Pietrocola, F., et al., Pro-autophagic polyphenols reduce the acetylation of cytoplasmic proteins. Cell Cycle, 2012. 11(20): p. 3851-60.

31. Yakhine-Diop, S.M., et al., Fluorescent FYVE Chimeras to Quantify Ptdlns3P Synthesis During Autophagy. Methods Enzymol, 2017. 587: p. 257-269.

32. Rodriguez-Arribas, M., et al., IFDOTMETER: A New Software Application for Automated Immunofluorescence Analysis. J Lab Autom, 2016. 21(2): p. 246-59.

33. Bolte, S. and F.P. Cordelieres, A guided tour into subcellular colocalization analysis in light microscopy. J Microsc, 2006. 224(Pt 3): p. 213-32.

34. Pfaffl, M.W., A new mathematical model for relative quantification in real-time RT-PCR. Nucleic Acids Res, 2001. 29(9): p. e45. 
35. Bravo-San Pedro, J.M., et al., The LRRK2 G2019S mutant exacerbates basal autophagy through activation of the MEK/ERK pathway. Cell Mol Life Sci, 2013. 70(1): p. 121-36.

36. Gomez-Sanchez, R., et al., Mitochondrial impairment increases FL-PINK1 levels by calcium-dependent gene expression. Neurobiol Dis, 2014. 62: p. 426-40.

37. Wei, X., et al., Cadmium induces mitophagy through ROS-mediated PINK1/Parkin pathway. Toxicol Mech Methods, 2014. 24(7): p. 504-11.

38. Thomas, K.J. and M.R. Jacobson, Defects in mitochondrial fission protein dynaminrelated protein 1 are linked to apoptotic resistance and autophagy in a lung cancer model. PLoS One, 2012. 7(9): p. e45319.

39. Min, S.W., et al., Sirtuins in neurodegenerative diseases: an update on potential mechanisms. Front Aging Neurosci, 2013. 5: p. 53.

40. Newman, J.C., W. He, and E. Verdin, Mitochondrial protein acylation and intermediary metabolism: regulation by sirtuins and implications for metabolic disease. J Biol Chem, 2012. 287(51): p. 42436-43.

41. Su, Y.C., X. Guo, and X. Qi, Threonine 56 phosphorylation of Bcl-2 is required for LRRK2 G2019S-induced mitochondrial depolarization and autophagy. Biochim Biophys Acta, 2015. 1852(1): p. 12-21.

42. Jin, H., et al., -Synuclein Negatively Regulates Protein Kinase C Expression to Suppress Apoptosis in Dopaminergic Neurons by Reducing p300 Histone Acetyltransferase Activity. Journal of Neuroscience, 2011. 31(6): p. 2035-2051.

43. Schiltz, R.L., et al., Overlapping but distinct patterns of histone acetylation by the human coactivators $p 300$ and PCAF within nucleosomal substrates. J Biol Chem, 1999. 274(3): p. 1189-92.

44. de Ruijter, A.J., et al., Histone deacetylases (HDACs): characterization of the classical HDAC family. Biochem J, 2003. 370(Pt 3): p. 737-49.

45. Kao, H.Y., et al., Isolation and characterization of mammalian HDAC10, a novel histone deacetylase. J Biol Chem, 2002. 277(1): p. 187-93.

46. Green, K.N., et al., Nicotinamide restores cognition in Alzheimer's disease transgenic mice via a mechanism involving sirtuin inhibition and selective reduction of Thr231phosphotau. J Neurosci, 2008. 28(45): p. 11500-10. 
47. Balasubramanyam, K., et al., Small molecule modulators of histone acety/transferase p300. J Biol Chem, 2003. 278(21): p. 19134-40.

48. Shi, S., et al., Dimeric structure of p300/CBP associated factor. BMC Struct Biol, 2014. 14: p. 2.

49. Huang, R., et al., Deacetylation of nuclear LC3 drives autophagy initiation under starvation. Mol Cell, 2015. 57(3): p. 456-66.

50. Nasrin, N., et al., JNK1 phosphorylates SIRT1 and promotes its enzymatic activity. PLoS One, 2009. 4(12): p. e8414.

51. Li, Y., et al., SirT1 inhibition reduces /GF-I/IRS-2/Ras/ERK1/2 signaling and protects neurons. Cell Metab, 2008. 8(1): p. 38-48.

52. Zhao, Y., et al., Interactions between SIRT1 and MAPK/ERK regulate neuronal apoptosis induced by traumatic brain injury in vitro and in vivo. Exp Neurol, 2012. 237(2): p. 489-98.

53. Dai, S.H., et al., Sirt3 protects cortical neurons against oxidative stress via regulating mitochondrial Ca2+ and mitochondrial biogenesis. Int J Mol Sci, 2014. 15(8): p. 14591-609.

54. Liu, L., et al., Protective role of SIRT5 against motor deficit and dopaminergic degeneration in MPTP-induced mice model of Parkinson's disease. Behav Brain Res, 2015. 281: p. 215-21.

55. Zhang, J.Y., et al., SIRT3 Acts as a Neuroprotective Agent in Rotenone-Induced Parkinson Cell Model. Neurochem Res, 2016. 41(7): p. 1761-73.

56. Yakhine-Diop, S.M.S., et al., Acetylome in Human Fibroblasts From Parkinson's Disease Patients. Frontiers in Cellular Neuroscience, 2018. 12(97).

57. Rouaux, C., et al., Critical loss of $C B P / p 300$ histone acetylase activity by caspase- 6 during neurodegeneration. EMBO J, 2003. 22(24): p. 6537-49.

58. Choong, C.J., et al., A novel histone deacetylase 1 and 2 isoform-specific inhibitor alleviates experimental Parkinson's disease. Neurobiol Aging, 2016. 37: p. 103-16.

59. Salama, A.F., et al., Epigenetic Study of Parkinsoni ${ }^{-}$s Disease in Experimental Animal Model. International Journal of Clinical and Experimental Neurology, 2015. 3(1): p. 11-20. 
60. More, S.V. and D.K. Choi, Emerging preclinical pharmacological targets for Parkinson's disease. Oncotarget, 2016. 7(20): p. 29835-63.

61. Schapira, A.H., et al., Mitochondrial complex I deficiency in Parkinson's disease. J Neurochem, 1990. 54(3): p. 823-7.

\section{Figure legends:}

Figure 1: Mitophagy regulation in cellular models of PD.

A-C/ HFs were transfected with GFP-LC3 plasmid and immunolabeled with TOM20 antibody. A/ Representative confocal images of transfected cells with GFP-LC3 (green) and co-labeled with TOM20 (red), Nuclei staining with DAPI (blue), Original magnification: $63 \mathrm{X}$, scale bar: $10 \mu \mathrm{m}$. The fifth column represents, as indicated by the profile $\alpha-\omega$, the distribution of fluorescence intensity in this section of the cell. $\mathbf{B} /$ Colocalization data analysis of TOM20 with GFP-LC3 using Manders' coefficient (ImageJ). Data are represented as the mean $\pm \mathrm{SD},{ }^{*} \mathrm{p}<0.05,{ }^{*} \mathrm{p}<0.01$ by Student's $\mathrm{t}-$ test. C/ Quantification of GFP-LC3 puncta per cell was determined by Ifdotmeter ${ }^{\circledR}$ software, $\left(\mathrm{n}=30\right.$ images/condition). Data are represented as the mean $\pm \mathrm{SD},{ }^{*} \mathrm{p}<0.05$, $* * p<0.01$ fold change to Co (Student's t-test). D, E/ Autophagy flux. HFs were treated with EBSS or BAF. A1 (100 nM) for 4 hours. The level of LC3 lipidation was assessed and normalized to GAPDH. Results are mean \pm SD of three independent experiments, ${ }^{*} \mathrm{p}<0.05$ and ${ }^{* *} \mathrm{p}<0.01$ versus untreated cells or between lines, (Student's t-test). F-H/ Cells were treated for 4 hours with CCCP $(10 \mu \mathrm{M})$ and/or CsA $(5 \mu \mathrm{M})$ and loaded with MTG fluorescence $(100 \mathrm{nM})$. Flow cytometry was used to assess mitophagy by determining the retention of MTG fluorescence. F/ Represents the analysis of flow cytometry data in treated and non-treated cells $(* * \mathrm{p}<0.01, * * * \mathrm{p}<0.001$ differences between lines) or $(+p<0.05,++p<0.01,+++p<0.001$ differences within line Co) or $(/ / \mathrm{p}<0.01, / / / \mathrm{p}<0.001$ differences within line IPD) or $(\$ \$ p<0.01, \$ \$ \$ p<0.001$ differences within line GS) (Student's t-test). Data are the mean \pm SD of three independents experiments. G/ Displays the basal difference in MTG retention between cell lines as depicted by the indicated histograms ( $n=10.000$ events). H/ Indicates the effect of CCCP (purple histogram) on MTG retention in line Co, basal condition (blue histogram).

Figure 2: Mitochondrial status and sirtuins expression in PD models. 
A-C/ Cells were maintained under basal conditions and co-stained with $\mathrm{DiOC}_{6}(3)$ (40 $\mathrm{nM})$ and dihydroetidium $(5 \mu \mathrm{M})$ or MitoSOX $(2 \mu \mathrm{M})$. The percentages of cells $\mathrm{DiOC}_{6}$ (3) low (A), Ethidium positive (B) and MitoSOX (C) positive were detected by flow cytometry. Data are the mean percentage $\pm \mathrm{SD} * \mathrm{p}<0.05,{ }^{* *} \mathrm{p}<0.01,{ }^{* * *} \mathrm{p}<0.001$ versus Co, (Student's t-test). D-F/ Mitochondrial markers. Cells were maintained under basal conditions. Expression levels of LONP1 (D, E) and DRP1 (D, F) were normalized to the loading control, GAPDH. Data correspond to the relative mean $\pm \mathrm{SD}$ of three independent experiments, ${ }^{*} \mathrm{p}<0.01$ versus Co, (Student's t-test). G/ Detection of PINK1 after 4h-CCCP $(10 \mu \mathrm{M})$ treatment in HFs. H/ Micrographs of HFs labeled with TOM20 antibody to determine mitochondrial morphology, white arrows display fragmented mitochondria. Original magnification: 40X, scale bar: $10 \mu \mathrm{m}$. I-K/ Expression of SIRT1 in HFs. I/ mRNA expression level of SIRT1 by qPCR. Data are the normalized mean $\pm \mathrm{SD}$ of three independents experiments $\left({ }^{*} \mathrm{p}<0.05\right.$ respect to $\left.\mathrm{Co}\right)$. J/ Assessment of pSIRT1 (serine 47) and SIRT1 by immunoblotting. K/ Level of pSIRT1 was normalized to total SIRT1. GAPDH is the loading control. Data are the relative mean $\pm \mathrm{SD}$ of three independent experiments, ${ }^{*} \mathrm{p}<0.05$ respect to Co, (Student's t-test). L-Q/ Expression of SIRT3 and SIRT5 in HFs. mRNA expression levels of SIRT3 (L) and SIRT5 (M) by qPCR. Data are the normalized mean \pm SD of three independent experiments, ${ }^{*} \mathrm{p}<0.05,{ }^{*} \mathrm{p}<0.01, * * * \mathrm{p}<0.001$ respect to Co, (Student's ttest). Detection of $\operatorname{SIRT5}(\mathbf{N}, \mathbf{P})$ and $\operatorname{SIRT3}(\mathbf{O}, \mathbf{Q})$ proteins by $\mathrm{WB}$ and their quantification is normalized to GAPDH. Data are the relative mean $\pm \mathrm{SD}$ of at least three independent experiments $* \mathrm{p}<0.05,{ }^{*} \mathrm{p}<0.01, * * * \mathrm{p}<0.001$ versus Co, (Student's ttest).

\section{Figure 3: Implication of SIRT1 in mitophagy}

A/ Detection of SIRT3 and TOM20 in HFs. B/ Detection of SIRT5, SIRT3, TOM20 and COX IV by immunoblotting in mitochondrial fraction of SH-SY5Y (WT and G20) maintained under basal conditions. SIRT5 and GAPDH were detected in cytosolic fraction. WT (SH-SY5Y WT LRRK2), G20 (SH-SY5Y G2019S LRRK2). C/ HFs were treated for 4 hours with CCCP $(10 \mu \mathrm{M})$ or CsA $(5 \mu \mathrm{M})$. D-J/ HFs were treated with nicotinamide (NAM, $1 \mathrm{mM})(\mathbf{D}-\mathbf{F})$ or EX-527 $(1 \mu \mathrm{M})(\mathbf{G}-\mathbf{J})$ for 4 hours. ATG5 (D, E and G, H), LC3 lipidation (D, F and G, I) and TOM20 (J) were detected by WB and quantified respect to the loading control, GAPDH. Results indicate the relative mean \pm $\mathrm{SD}$ of three independents experiments, $\# \mathrm{p}>0.05,{ }^{*} \mathrm{p}<0.05,{ }^{*} \mathrm{p}<0.01$ versus Co, (Student's t-test). kDa (kilodaltons). K/ Genetic inhibition of SIRT1 in HFs. SIRT1, SIRT3 and TOM20 were detected by immunoblotting, GAPDH is the loading control. L/ HFs were treated with EX-527 $(1 \mu \mathrm{M})$ for 4 hours and loaded with MTG (100 nM) to detect mitochondria, white arrows show mitochondrial fragmentation. MTG staining was observed by in vivo immunofluorescence. Original magnification: 20X, scale bar: $10 \mu \mathrm{m}$.

Figure 4: Acetylated lysine protein levels in cellular models of PD. 
Acetylated proteins. A/ WB analyses show the difference in acetylated proteins between HFs (Co, IPD and GS) and neuroblastoma SH-SY5Y (WT and G20) using antibodies against acetylated lysine (Ac-K) and GAPDH as a loading control. The upper blot is less exposed and the lower blot is higher exposed. WT (SH-SY5Y WT LRRK2), G20 (SH-SY5Y G2019S LRRK2). B, C/ Immunofluorescence intensity of labeled cytoplasmic Ac-K proteins (red) in HFs, Original magnification: 40X, scale bar corresponds to $10 \mu \mathrm{m}$. C/ Represents the quantification of the fluorescence intensity $(n=200$ cells/condition). Data are the mean \pm SEM of three independent experiments, $*^{*} \mathrm{p}<0.01$ in comparison to Co, (Student's t-test). D-F/ Cells were incubated with fresh medium or EBSS for $4 \mathrm{~h}$. D/ LC3 lipidation is determined by WB and its densitometry is the ratio of LC3-II/I. GAPDH is the loading control. E/ Immunofluorescence intensity of endogenous Ac-K proteins (red) in SH-SY5Y, Original magnification: 20X, scale bar: $10 \mu \mathrm{m} . \mathbf{F} /$ Represents the quantification of fluorescence intensity of labeled Ac-K proteins ( $n=15$ images/condition). Data represent the mean $\pm \mathrm{SD}$ of at least three independent experiments, ${ }^{*} \mathrm{p}<0.05, * * \mathrm{p}<0.01$ versus WT or untreated conditions, (Student's t-test). WT (SH-SY5Y WT LRRK2), G20 (SH-SY5Y G2019S LRRK2). MW (molecular weight), $\mathrm{kDa}$ (kilodaltons). Histone acetyltransferase activity and expression levels. G-K/ HATs proteins, such as TIP60 (G, H) and PCAF (J, K), were detected by WB. Their densitometry was referenced to GAPDH. The results correspond to the mean $\pm \mathrm{SD}$ of three independent experiments, $* \mathrm{p}<0.05, * * \mathrm{p}<0.01$ versus Co, (Student's ttest). I/ Assessment of HATs activity by colorimetry. The results are in absorbance/ $\mu \mathrm{g}$ proteins $(\lambda=400 \mathrm{~nm})$ and represent the mean \pm SD of three independent experiments. WT (SH-SY5Y WT LRRK2), G20 (SH-SY5Y G2019S LRRK2). L-O/ Detection of acetylated Histone 4 on lysine 5, 8, 12 and phosphorylated on serine 1(Ac-H4 or AcH4Ser1K5K8K12) (L, M) and acetylated tubulin on lysine 40 (Ac-Tubk40) (N, O) by immunoblotting and normalized to total Histone 3 (H3) and $\alpha$-tubulin, respectively. Data are the mean $\pm \mathrm{SD}$ of at least three independent experiments, ${ }^{*} \mathrm{p}<0.05, * * * \mathrm{p}<0.001$ compared to Co, (Student's t-test). kDa (kilodaltons), *un (unspecific band of Ac-H4).

\section{Figure 5: Histone deacetylase activity and expression levels.}

A-I/ Detection of the expression levels of HDAC2 (A, B), HDAC3 (C, D), HDAC4 (E, $\mathbf{F})$, HDAC1 (E, G), HDAC6 $(\mathbf{H}, \mathbf{I})$ and their densitometry normalized to the loading control, GAPDH. Data represent the relative mean $\pm \mathrm{SD}$ of at least three independent experiments, $* * p<0.01$ and $* * * p<0.001$ respect to Co, (Student's t-test). $\mathrm{kDa}$ (kilodaltons). $\mathbf{J} /$ Results show the colorimetric HDAC activity in absorbance/ $\mu \mathrm{g}$ proteins $(\lambda=405 \mathrm{~nm})$. Data are the mean \pm SD of three independent experiments, $* * * \mathrm{p}<0.001$ versus Co, (Student's t-test). WT (SH-SY5Y WT LRRK2), G20 (SH-SY5Y G2019S LRRK2). Effects of Histone deacetylase inhibitors, TSA and NAM. K/ HFs were treated with TSA $(1 \mu \mathrm{M})$ and NAM $(1 \mathrm{mM})$ for $4 \mathrm{~h}$. Histone 4 acetylated on lysine 16 (Ac-H4K16) (green) was detected by immunofluorescence and the nuclei were stained with Hoechst 33342 (blue), Original magnification: 40X scale bar corresponds to $10 \mu \mathrm{m}$. L/ Cells were treated for $15 \mathrm{~h}$ with TSA $(1 \mu \mathrm{M})$ or NAM $(1 \mathrm{mM})$ and stained with Annexin V-FITC. The results represent the percentage \pm SD of apoptotic cells 
detected by flow cytometry $(* * \mathrm{p}<0.01, * * * \mathrm{p}<0.001$ in comparison to $\mathrm{Co}$, in basal condition) or $(++\mathrm{p}<0.01,+++\mathrm{p}<0.001$ within Co line $)$ or $(/ / \mathrm{p}<0.01$ within IPD line $)$ or $(\$ \$ p<0.01$ within GS), (Student's t-test). M, N/ Cells were treated for $4 \mathrm{~h}$ with TSA (1 $\mu \mathrm{M})$ or NAM $(1 \mathrm{mM})$ or CCCP $(10 \mu \mathrm{M})$ and then loaded with TMRM (50 $\mathrm{nM})$, Original magnification: 40X, scale bar: $10 \mu \mathrm{m}$. The intensity of TMRM was observed by in vivo immunofluorescence $(\mathrm{n}=30)$, the results represent the mean percentage \pm SD $(* * p<0.01, * * * p<0.001$ versus Co, in basal condition) or $(+++p<0.001$ within Co line) or $(/ \mathrm{p}<0.05, / / \mathrm{p}<0.01, / / / \mathrm{p}<0.001$ within IPD line) or ( $\$ \mathrm{p}<0.01$ within GS), (Student's ttest).

\section{Figure 6: Effect of anacardic acid on PD models.}

A, B/ HFs were treated with $10 \mu \mathrm{M}$ anacardic acid (AA) for one h. Histone 3 acetylated on lysine 14 (Ac-H3K14) was detected by immunoblotting, and it was normalized to total histone 3. Data are the mean $\pm \mathrm{SD}$ at least of three independent experiments ${ }^{*} \mathrm{p}<0.05$ versus Co or untreated conditions or between treated cells, (Student's t-test). C/ HFs were treated for $15 \mathrm{~h}$ with $10 \mu \mathrm{M}$ AA. D/ WT (SH-SY5Y WT LRRK2) were pretreated for one h with AA $(10 \mu \mathrm{M})$ and afterwards incubated with $\mathrm{MPP}^{+}(500 \mu \mathrm{M})$ for $15 \mathrm{~h}$. Next, both lines were stained with propidium iodide (PI), and the percentage of PI-positive $\left(\mathrm{PI}^{+}\right)$cells was evaluated by flow cytometry, $(\mathrm{n}=10.000$ events $)$. Data are the mean percentage \pm SD of three independent experiments, ${ }^{*} \mathrm{p}<0.05$ compared to Co or untreated cells or between treated cells, (Student's t-test). Relevant differences between PD models. E/ The schema highlights the differences that occur between idiopathic and genetic PD patients. In idiopathic PD patients, mitophagy downregulation heightens ROS generation and cell death through the inhibition of SIRT activity. Although ROS is increased in genetic PD patients, its consequences are reduced due to enhanced mitochondrial turnover related to SIRT1 increase.

\section{Figure S1: Determination of G2019S LRRK2 mutation and expression levels of SIRT2 and SIRT6.}

A/ Restriction enzyme of LRRK2 exon 41. Bfm I hydrolyses the exon 41 harboring the G2019S mutation into 2 bands (300 and 200 base pairs (bp)) confirming that the mutation is heterozygous. SIRT2 expression levels. B/ mRNA expression of SIRT2 by qPCR. Data are the normalized mean \pm SD of three independent experiments. C-E/ Detection of two isoforms of SIRT2 by immunoblotting, SIRT2 isoform I (43 kDa) (C, D) and SIRT2 isoform II (39 kDa) (C, E). The densitometry of each isoform is normalized to GAPDH. The results correspond to the relative mean $\pm \mathrm{SD}$ of three independent experiments, ${ }^{*} \mathrm{p}<0.05$ in comparison to Co, (Student's t-test). F-H/ SIRT6 expression levels. F/ mRNA expression of SIRT6 by qPCR. Data are the normalized mean $\pm \mathrm{SD}$ of three independent experiments, ${ }^{* *} \mathrm{p}<0.01$ versus Co, (Student's t-test). G, $\mathbf{H} /$ Assessment of SIRT6 expression and its quantification to referenced GAPDH. Data are the normalized means $\pm \mathrm{SD}$ of three independent experiments, $* * \mathrm{p}<0.01$ up to Co, (Student's t-test).

Figure S2: mRNA expression levels of the HAT family. 
A, B/ Immunofluorescence intensity of labeled nuclear Ac-K proteins (red) in HFs, the nuclei were stained with Hoechst 33342 (blue). Original magnification: 40X, scale bar corresponds to $10 \mu \mathrm{m}$. B/ Represents the quantification of the fluorescence intensity $(\mathrm{n}=60$ cells/condition). Data are the mean \pm SEM of two independent experiments, $* * \mathrm{p}<0.01, * * * \mathrm{p}<0.001$ in comparison to Co, (Student's t-test). p300 (C) and PCAF (D) are members of the p300/CBP and GNAT families, respectively. Members of the MYST family are hMOF (E) and TIP60 (F). Their mRNA expression levels were assessed by $\mathrm{qPCR}$, and the results represent the relative mean $\pm \mathrm{SD}$ of at least three independent experiments, ${ }^{*} \mathrm{p}<0.05,{ }^{*} \mathrm{p}<0.01,{ }^{* *} \mathrm{p}<0.001$ versus $\mathrm{Co}$, (Student's t-test). G/ Immunofluorescence intensity of labeled cytoplasmic Ac-K proteins (red) in HFs treated with TSA $(1 \mu \mathrm{M})$ during 4 hours. Scale bar corresponds to $10 \mu \mathrm{m}$.

\section{Figure S3: mRNA expression levels of class I and II HDACs.}

A-C/ Class I HDACs includes HDAC1 (A), HDAC2 (B) and HDAC3 (C). D, E/ Class II HDACs includes HDAC4 (D) and HDAC6 (E). Their mRNA expression levels were assessed by qPCR, and the results correspond to the relative mean \pm SD of three independent experiments, ${ }^{*} p<0.05,{ }^{*} p<<0.01,{ }^{* * *} p<0.001$ versus Co, (Student's t-test).

\section{Figure S4: Cell viability with HDAC inhibitors.}

A, B/ HFs were treated with TSA $(0-100 \mu \mathrm{M})$ or NAM $(0-100 \mathrm{mM})$ for $24 \mathrm{~h}$. Cell viability was assessed by the colorimetric test MTT. Data correspond to the normalized mean percentage of untreated cells, ${ }^{*} \mathrm{p}<0.05,{ }^{* *} \mathrm{p}<0.01,{ }^{* * *} \mathrm{p}<0.001$, (Student's t-test). C, D/ Cells were treated with TSA $(1 \mu \mathrm{M})$ or NAM $(1 \mathrm{mM})$ for $4 \mathrm{~h}$. Histone 3 acetylated on lysine 14 (Ac-H3K14) was detected by immunoblotting and was normalized to total Histone 3 (H3). E/ Cells were treated overnight with EX-527 (1 $\mu \mathrm{M})$. Cells were stained with propidium iodide (PI), and the percentage of $\mathrm{PI}^{+}$cells was evaluated by flow cytometry, $(n=10.000$ events $)$. Data are the mean percentage \pm SD of three independent experiments, ${ }^{*} p<0.05,{ }^{* *} p<0.01$, (Student's t-test).

\section{Abbreviations:}

AA: Anacardic acid, Ac-K: Acetylated lysine, ATG: Autophagy-related, BAF. A1: Bafilomycin A1, CBP: CREB-binding protein, CCCP: Carbonyl cyanide 3chlorophenylhydrazone, COX IV: Cytochrome c oxidase subunit 4, CsA: Cyclosporin A, DiOC6(3): 3,3'-dihexyloxacarbocyanine iodide, DRP-1: Dynamin related protein 1, EBSS: Earl's Balanced Salt Solution, ERK1/2: Extracellular signalregulated kinase 1/2, GAPDH: Glyceraldehyde-3-phosphate dehydrogenase, GNAT: GCN5-related N-acetyltransferase, GFP: Green fluorescent protein, H3: Histone 3, H3K14: Histone 3 lysine 14, H4: Histone 4, H4K5K8K12: Histone 4 lysine 5 lysine 8 lysine 12, H4K16: Histone 4 lysine 16. HAT: Histone acetyltransferase, HDAC: Histone deacetylase, hMOF: Human male absent of first, IPD: Idiopathic Parkinson's disease, JNK1: Jun-N-terminal kinase 1, LC3: Light-chain microtubule-associated protein, LONP1: Lon peptidase 1, LRRK2: Leucine-rich repeat kinase 2, MAPK: Mitogen-activated protein kinase, MMP: Mitochondrial membrane potential, $\mathrm{MPP}^{+}: 1-$ Methyl-4-phenylpyridinium iodide, MPTP: 1-Methyl-4-phenyl-1,2,3,6tetrahydropyridine hydrochloride, MTG: MitoTracker green, mTOR: Mammalian target 
of rapamycin, $\mathrm{NAD}^{+}$: Nicotinamide adenine dinucleotide, NAM: Nicotinamide, PCAF: p300/CREB-binding protein-associated factor, PD: Parkinson's disease, PI: Propidium iodide, PINK1: PTEN-induced putative kinase 1, ROS: Reactive oxygen species, RT: Room temperature, SIRT: Sirtuin, TIP60: Tat-interactive protein 60, TMRM: Tetramethylrhodamine methyl ester perchlorate, TSA: Trichostatin A, WT: Wild-type 


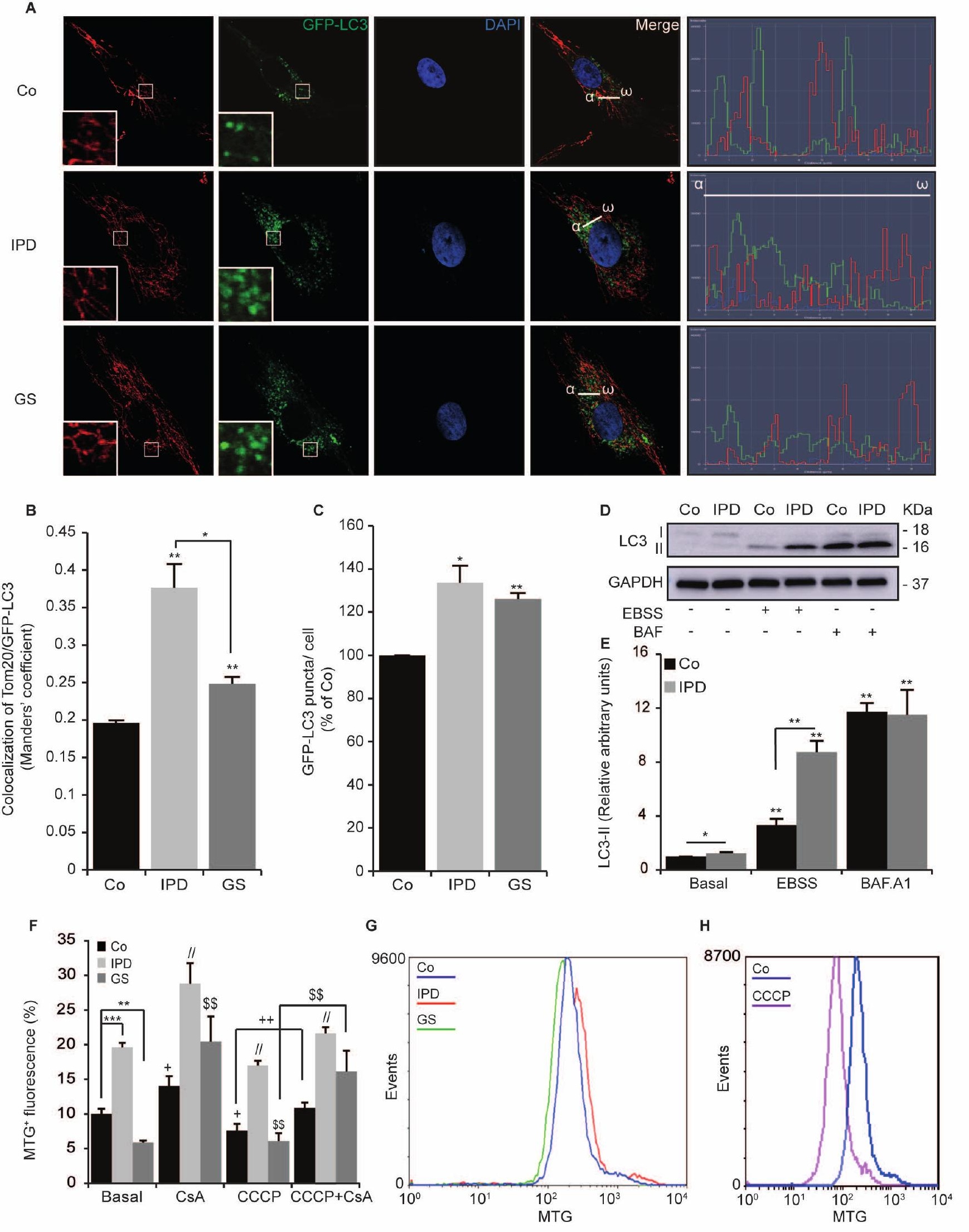

Figure 1 


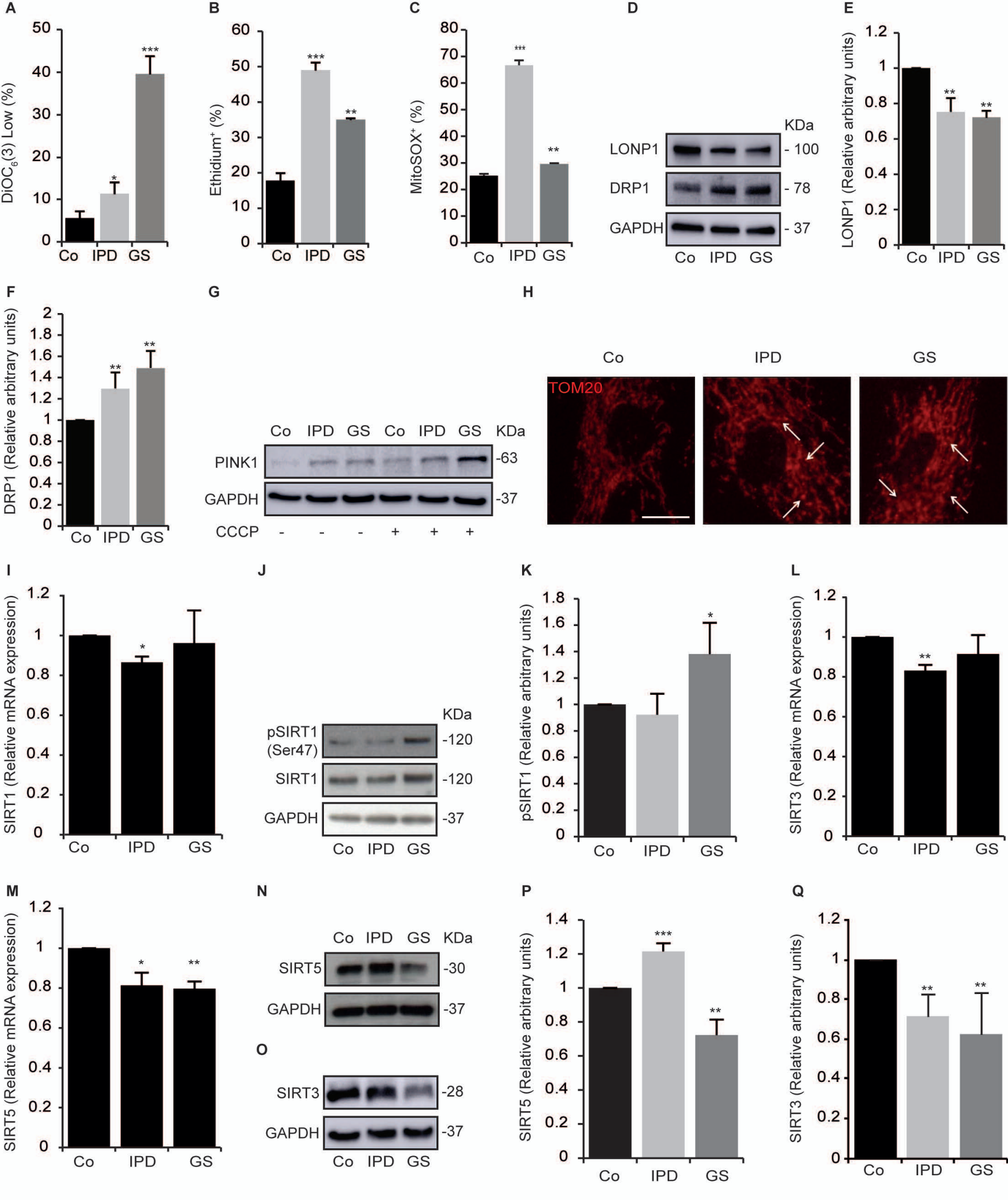





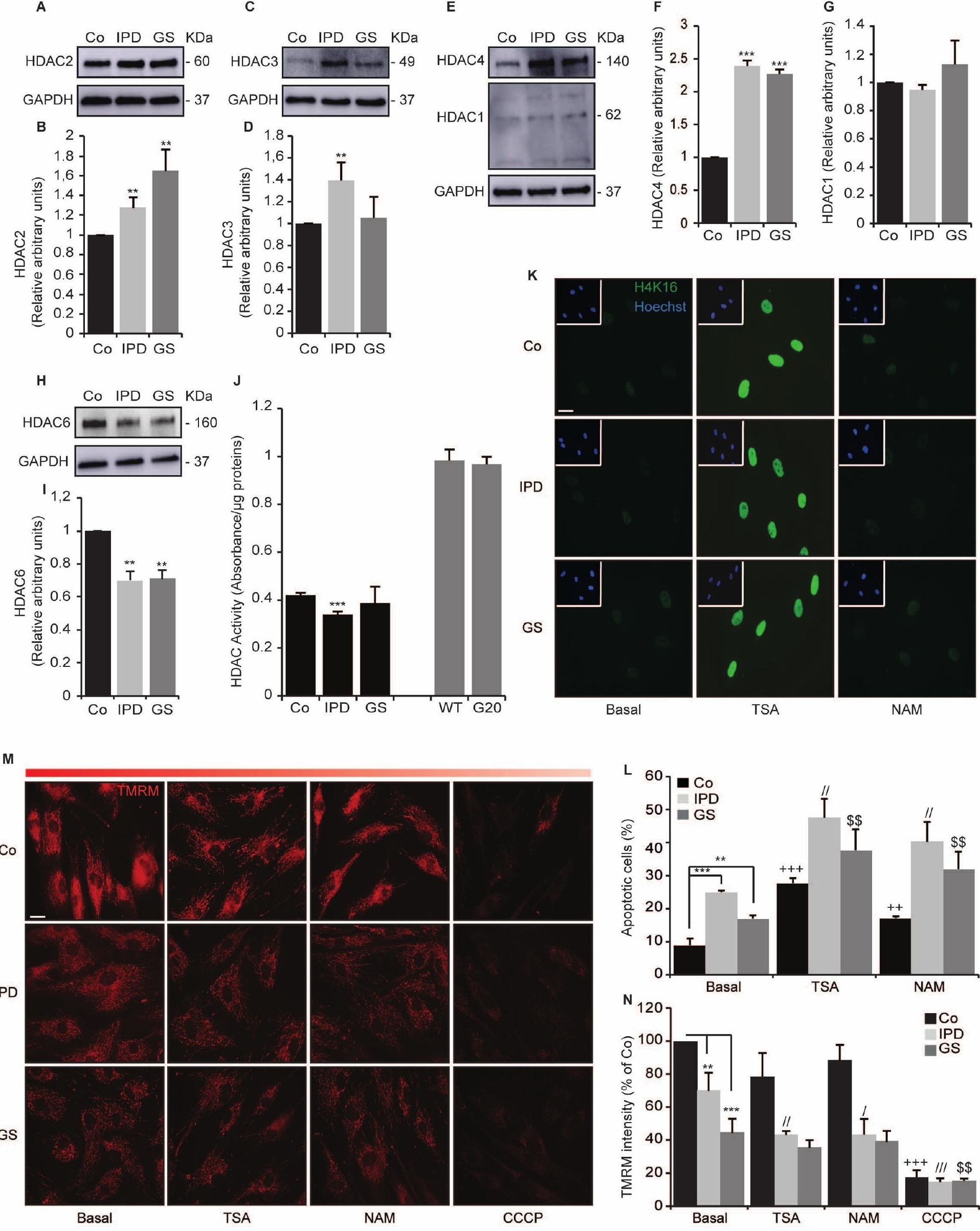

Figure 5 

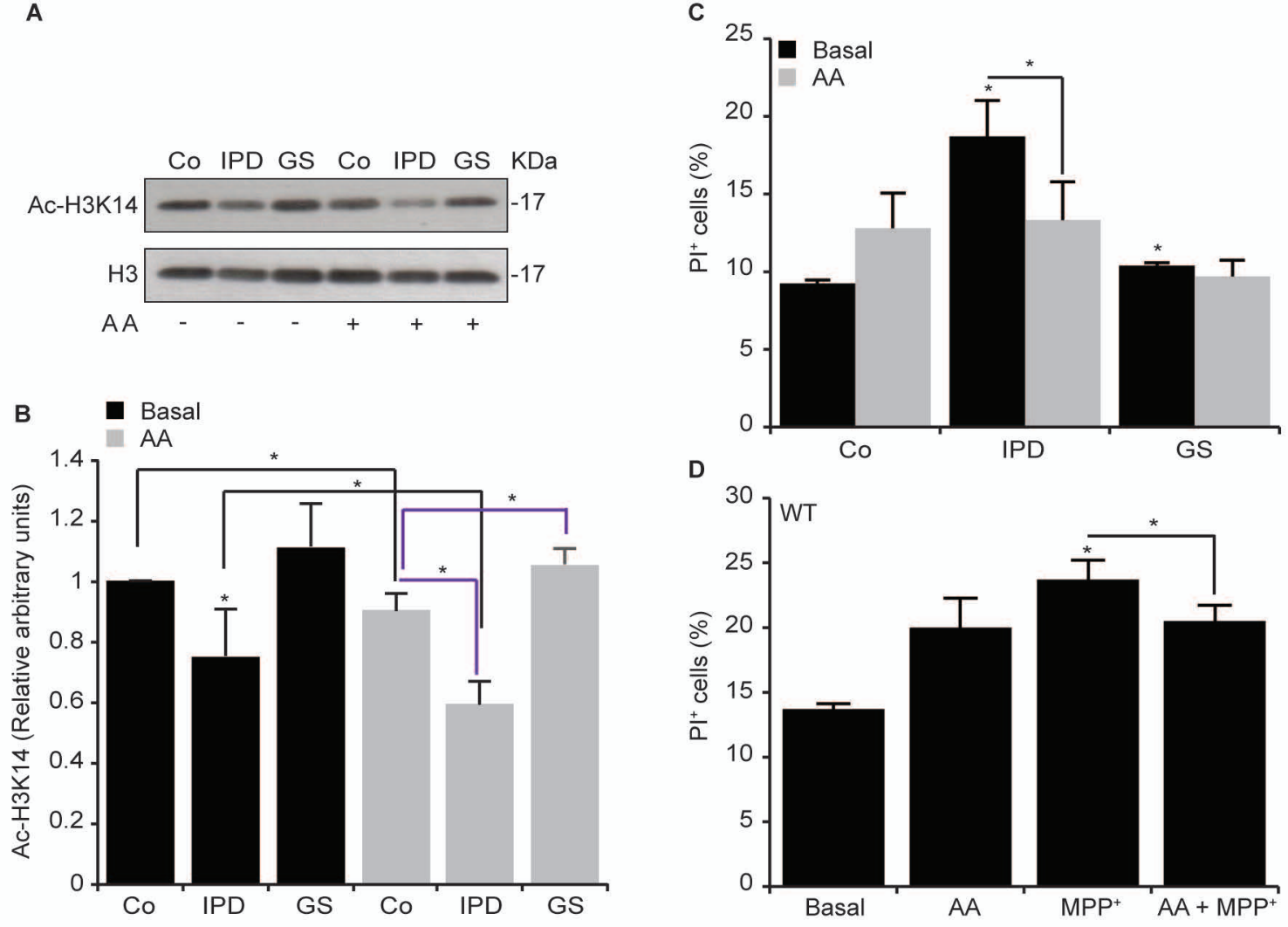

E
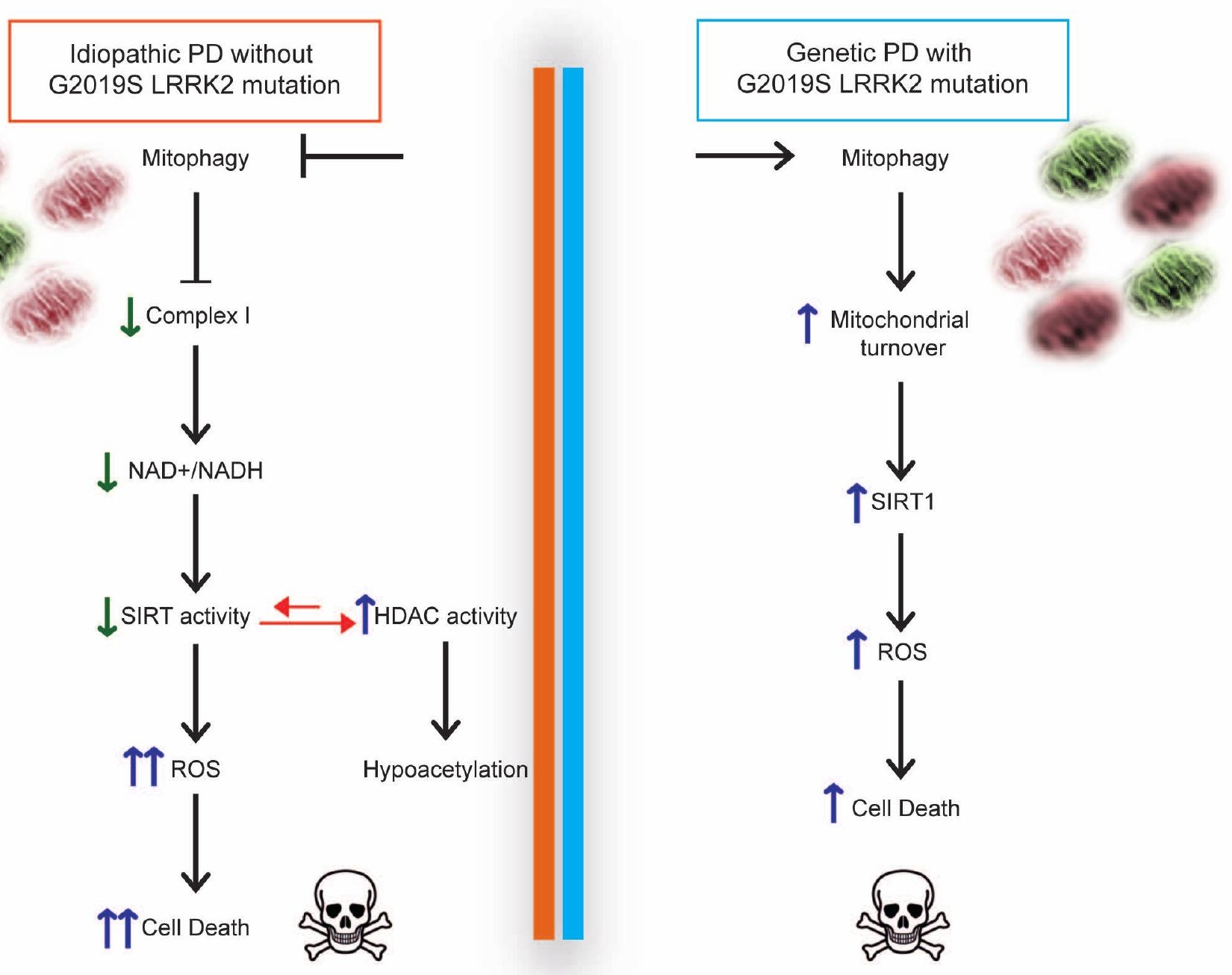
turnover
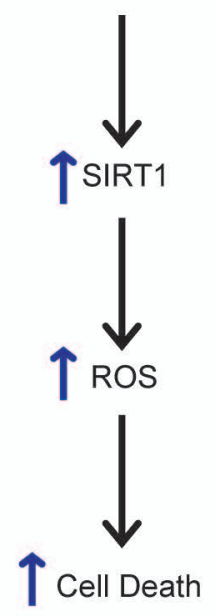


\begin{tabular}{|l|l|l|}
\hline Classes & Subclasses & \multicolumn{1}{|c|}{ Members } \\
\hline Class I & & HDAC1, HDAC2, HDAC3, HDAC8 \\
\hline Class II & Class IIa & HDAC4, HDAC5, HDAC7, HDAC9 \\
\cline { 2 - 3 } & Class IIb & HDAC6, HDAC10 \\
\hline Class III & & SIRT1, SIRT2, SIRT3, SIRT4, SIRT5, SIRT6, SIRT7 \\
\hline Class IV & & HDAC11 \\
\hline
\end{tabular}

Supplementary Table 1: Classification of HDACs and their respective members. This table lists the 18 HDAC proteins that are classified into four classes depending on their structure and homology shared with yeast proteins. 


\begin{tabular}{|c|c|c|c|c|}
\hline Groups & Names & Date of Birth & Genotype & Sexe \\
\hline \multirow{4}{*}{ Co } & Co1 & \multirow{4}{*}{$1956-1977$} & LRRK2 WT & $\hat{0}$ \\
\hline & $\mathrm{Co} 2$ & & LRRK2 WT & q \\
\hline & Co3 & & LRRK2 WT & क \\
\hline & $\mathrm{Co} 4$ & & LRRK2 WT & q \\
\hline \multirow{3}{*}{ IPD } & IPD1 & \multirow{3}{*}{$1928-1954$} & LRRK2 WT & q \\
\hline & IPD2 & & LRRK2 WT & $0^{\lambda}$ \\
\hline & IPD3 & & LRRK2 WT & q \\
\hline \multirow{3}{*}{ GS } & GS1 & \multirow{3}{*}{ 1945-1949 } & G2019S Heterozygous & $\hat{0}$ \\
\hline & GS2 & & G2019S Heterozygous & q \\
\hline & GS3 & & G2019S Heterozygous & q \\
\hline
\end{tabular}

Suplementary Table 2: Presentation of the three groups of individuals. The control group consists of four individuals, the IPD and GS groups of three individuals each. We present in this table the age, sex and genotype of each individual. 